\title{
Hematological Indices in Controlled and Uncontrolled Type 2 Diabetes Mellitus
}

\author{
Swathi Prakash ${ }^{1}$, Usha Setty Muthihar Ramachary ${ }^{2}$, Sharmila Surhonne Prakash ${ }^{3}$
}

\begin{abstract}
Aim and objective: To compare HbA1c levels with inflammatory markers that include a neutrophil-to-lymphocyte ratio (NLR) and monocyteto-lymphocyte ratio (MLR) in controlled and uncontrolled diabetics.

Materials and methods: Eighty-nine patients with type 2 diabetes mellitus (T2DM) were divided into two groups of controlled (HbA1c $<7 \%$ ) and uncontrolled (HbA1c $>7 \%$ ) diabetics. Recent laboratory data were used to collect HbA1c (glycated hemoglobin) levels, leukocyte count (WBC), hemoglobin $(\mathrm{Hb})$, hematocrit $(\mathrm{Hct})$, red blood cell distribution width (RDW), neutrophils, lymphocytes, and monocytes. The NLR and MLR were calculated from the laboratory data.

Results: The mean age in controlled and uncontrolled diabetics was 58.30 and 55.62 years, respectively. The mean NLR in controlled and uncontrolled diabetics was 2.61 and 4.88. The difference was found to be statistically significant $(p<0.05)$. The mean MLR in controlled and uncontrolled diabetics was 0.2 and 0.24 , but the difference was not statistically significant $(p>0.05)$. A weak positive correlation was found between $\mathrm{HbA} 1 \mathrm{c}$ levels and the hematological indices and the results were statistically insignificant.

Conclusion: The study yielded significant results in the difference between controlled and uncontrolled diabetics with respect to NLR. Although there was only a weak positive correlation found between glycated $\mathrm{Hb}$ levels and the hematological indices, the results showed that there is a significant difference in the NLR between the two groups. This proves that there is scope for use of these ratios as inflammatory markers in T2DM.

Keywords: Diabetes, Diabetes mellitus, Hematological indices, Inflammation, Inflammatory markers, Microvascular complications, Monocyteto-lymphocyte ratio, Neutrophil-to-lymphocyte ratio, Type 2 diabetes mellitus.

The Journal of Medical Sciences (2020): 10.5005/jp-journals-10045-00146
\end{abstract}

\section{INTRODUCTION}

The global prevalence of diabetes mellitus has been rapidly rising from $4.7 \%$ in 1980 to $8.5 \%$ in 2014 making it the 6 th leading cause of death worldwide. ${ }^{1}$ Type 2 diabetes mellitus (T2DM) is a metabolic disorder characterized by chronic hyperglycemia due to insulin resistance leading to several microvascular and macrovascular complications. Many articles have inferred that an inflammatory response is a likely contributor to insulin resistance and is intensified by chronic hyperglycemia which further aggravates the various complications of diabetes mellitus. ${ }^{2,3}$ Diabetes and uncontrolled hyperglycemia are known to play a significant role in the development of cardiovascular disease since the Framingham study. The presence of microvascular complications may lead to coronary events. ${ }^{4}$

In recent years, there has been extensive research on potential systemic inflammatory markers such as neutrophil-to-lymphocyte ratios (NLR) and monocyte-to-lymphocyte ratios (MLR) in various diseases like tumors, ${ }^{5-8}$ cardiovascular conditions, ${ }^{9,10}$ and other diseases. ${ }^{11}$ An increase in these markers are designated as predictors of endothelial dysfunction and inflammation. ${ }^{12-20}$

Over the past 5 years, the NLR has risen to popularity owing to its prognostic value. An increase in NLR is a poor clinical indicator in COVID-19 disease, ${ }^{21}$ lung cancer, ${ }^{22}$ etc. Endogenous cortisol and catecholamines may be major drivers of the NLR. Increased levels of cortisol are known to increase the neutrophil count while simultaneously decreasing the lymphocyte count. ${ }^{23}$ An increase in the MLR is another novel inflammatory marker that could possibly detect vascular disease or complications. Monocytes and monocyte-derived macrophages play important roles in the initiation and progression of atherosclerotic disease. ${ }^{24-26}$
${ }^{1}$ RajaRajeswari Medical College and Hospital, Bengaluru, Karnataka, India

${ }^{2}$ Department of Biochemistry, RajaRajeswari Medical College and Hospital, Bengaluru, Karnataka, India

${ }^{3}$ Department of Pathology, RajaRajeswari Medical College and Hospital, Bengaluru, Karnataka, India

Corresponding Author: Usha Setty Muthihar Ramachary, Department of Biochemistry, RajaRajeswari Medical College and Hospital, Bengaluru, Karnataka, India, Phone: +919945698453, email: usha_ manohar@yahoo.com

How to cite this article: Prakash S, Muthihar Ramachary US, Surhonne Prakash S. Hematological Indices in Controlled and Uncontrolled Type 2 Diabetes Mellitus. J Med Sci 2020;6(2):28-31.

Source of support: Nil

Conflict of interest: None

Diabetes and hyperglycemia create a proinflammatory microenvironment that progresses to microvascular complications, such as, nephropathy, retinopathy, and neuropathy. ${ }^{27}$ Obesity is a major risk factor for diabetes and can induce inflammation by toll-like receptor (TLR) activation to recruit proinflammatory cytokines and chemokines. ${ }^{28}$ Proinflammatory cytokines, C-reactive protein, tumor necrosis factor (TNF)- $a$, and interleukin (IL)- 6 have all demonstrated increased expression in diabetes. ${ }^{29}$ To date, there have been only a few articles that have studied the relationship between diabetes mellitus and these systemic inflammatory markers. $^{13-17}$ 
We hypothesize that there will be an increase in NLR and MLR while comparing uncontrolled diabetics to controlled diabetics. The goal of our study was to evaluate the differences in NLR and MLR between controlled and uncontrolled T2DM along with correlating $\mathrm{HbA} 1 \mathrm{c}$ with NLR and MLR.

Neutrophil-to-lymphocyte ratios and MLR are inexpensive, routinely done, and easily available markers of inflammation. Establishing a concrete association between the hematological indices and $\mathrm{HbA1c}$ would help in the utility of these markers in predicting complications of T2DM. Hence, explaining the need for the study.

\section{Materials and Methods}

The study protocol was approved by the Institutional Ethics Committee of RajaRajeswari Medical College and Hospital on $21 / 07 / 2018$. Data were collected from the patients who came to the hospital for follow-up. Forty-three patients served as controlled diabetics ( $\mathrm{HbA1c}<7 \%$ ), out of which only 39 patients fulfilled the inclusion criteria. Fifty patients served as uncontrolled diabetics (HbA1c $>7 \%)$. Subjects $<25$ years and with a history of hepatic failure, acute illness, cancer, and type 1 diabetes mellitus were excluded from the study.

A patient file search was performed to obtain information on $\mathrm{HbA1c}$ levels, leukocyte count (WBC), hemoglobin ( $\mathrm{Hb})$, hematocrit (Hct), red blood cell distribution width (RDW), neutrophils, lymphocytes, and monocytes. Ratios like NLR and MLR were calculated from the laboratory data.

\section{Statistical Analysis}

Statistical analyses were performed using Statistical Package for the Social Sciences (SPSS) 22.0 software pack. For data assessment, an independent $t$-test was used for the comparison of hematological indices in controlled diabetics vs uncontrolled diabetics. In addition, the Karl Pearson correlation test was performed to compare $\mathrm{HbA1c}$ levels with the hematological indices. The $t$-test results were expressed with $95 \%$ confidence intervals and a $p$ value. The Karl Pearson correlation test results were expressed with an $R$ value and a $p$ value. A $p$ value of $<0.05$ was considered significant.

\section{Results}

A total of 89 diabetic patients were stratified into controlled diabetics (HbA1c $<7 \%, n=39$, mean age $58.30 \pm 12.02$ ) and uncontrolled diabetics (HbA1c $>7 \%, n=50$, mean age $55.62 \pm 11.14$ ). The mean $\mathrm{Hb}, \mathrm{Hct}$, and RDW levels were comparable between the groups. The mean $\mathrm{HbA} 1 \mathrm{c}$ levels were $6.02 \pm 0.50 \%$ in controlled T2DM and $11.52 \pm 2.31 \%$ in uncontrolled T2DM. A comparison was drawn between the two groups with respect to demographic and laboratory data using a two-sample $t$-test in Table 1. There were statistically significant differences $(p<0.05)$ between the groups with respect to the following variables, such as, HbA1c levels, WBC count, neutrophil count, and NLR. There were no statistically significant differences found between the groups with respect to the following variables, such as, age, Hb, Hct, RDW, lymphocyte count, monocyte count, and MLR.

Using the Karl Pearson correlation test, a correlation test was done between $\mathrm{HbA1c}$ values and the hematological indices which include NLR and MLR in Table 2. It was found that there was a weak positive correlation while comparing $\mathrm{HbA1c}$ against NLR and MLR. These results were found to be statistically insignificant.
Table 1: Demographic and laboratory data of controlled and uncontrolled diabetics

\begin{tabular}{llll}
\hline Parameters & $\begin{array}{l}\text { HbA1c }<7 \\
\text { (controlled) } n=39\end{array}$ & $\begin{array}{l}\text { HbA1c }>7 \\
\text { (uncontrolled) } n=50\end{array}$ & p value \\
\hline Age (years) & $58.30 \pm 12.02$ & $55.62 \pm 11.14$ & 0.28 \\
HbA1c (\%) & $6.02 \pm 0.50$ & $11.52 \pm 2.31$ & $<0.01^{*}$ \\
WBC $\left(10^{3} / \mu \mathrm{L}\right)$ & $8.27 \pm 2.28$ & $10.34 \pm 3.78$ & $0.002^{*}$ \\
$\begin{array}{l}\text { Hemoglobin } \\
\text { (g/dL) }\end{array}$ & $12.72 \pm 2.85$ & $12.40 \pm 2.43$ & 0.58 \\
Hematocrit (\%) & $42.04 \pm 8.73$ & $40.78 \pm 7.36$ & 0.47 \\
RDW (\%) & $14.76 \pm 2.02$ & $13.81 \pm 2.46$ & 0.051 \\
$\begin{array}{l}\text { Neutrophil } \\
\left(10^{3} / \mu L\right)\end{array}$ & $5.15 \pm 1.85$ & $7.22 \pm 4.09$ & $0.002^{*}$ \\
Lymphocyte & $2.22 \pm 0.93$ & $2.33 \pm 0.88$ & 0.58 \\
$\left(10^{3} / \mu \mathrm{L}\right)$ & & & \\
Monocyte $\left(10^{3} /\right.$ & $0.44 \pm 0.16$ & $0.48 \pm 0.20$ & 0.34 \\
$\mu \mathrm{L})$ & & & \\
NLR & $2.61 \pm 1.31$ & $4.11 \pm 4.64$ & $0.03^{*}$ \\
MLR & $0.2 \pm 0.08$ & $0.24 \pm 0.14$ & 0.27 \\
\hline
\end{tabular}

${ }^{*} p$ value $<0.05$ suggests strong statistical significance using independent $t$-test

NLR, neutrophil-to-lymphocyte ratio; MLR, monocyte-to-lymphocyte ratio; RDW, red blood cell distribution width

Table 2: Correlation between $\mathrm{HbA} 1 \mathrm{c}$ and hematological indices

\begin{tabular}{lll}
\hline HbA1c vs parameters & $R$ value & $p$ value* \\
\hline HbA1c vs NLR & +0.062 & 0.55 \\
HbA1c vs MLR & +0.031 & 0.89 \\
\hline
\end{tabular}

${ }^{*} p$ value $<0.05$ suggests strong statistical significance using the Karl Pearson correlation test

NLR, neutrophil-to-lymphocyte ratio; MLR, monocyte-to-lymphocyte ratio

\section{Discussion}

The NLR and MLR are relatively newer, simpler, and inexpensive laboratory markers that can be readily estimated. The association between these hematological parameters and many medical pathologies have been clearly established. ${ }^{18}$

The goal of our study was to compare NLR and MLR ratios among controlled and uncontrolled diabetics and then correlate the $\mathrm{HbA1c}$ values with these ratios. The mean WBC and neutrophil counts were found to be higher among uncontrolled diabetics with the difference being statistically significant when compared with controlled diabetics.

Higher NLR values $(4.11 \pm 4.64$ vs $2.61 \pm 1.31)$ were found among uncontrolled diabetics when compared with controlled diabetics, respectively. The difference was found to be statistically significant. Higher MLR values $(0.24 \pm 0.14$ vs $0.2 \pm 0.08)$ were found while comparing uncontrolled diabetics $(\mathrm{HbA} 1 \mathrm{c}>7 \%)$ to controlled diabetics ( $\mathrm{HbA1c}<7 \%$ ), respectively. This difference was found to be statistically insignificant.

A Karl Pearson correlation test was conducted between $\mathrm{HbA1c}$ values vs NLR and MLR. There was a weak positive correlation found between the HbA1c levels and the hematological indices. However, the results were statistically insignificant.

Patients with T2DM are at high risk for microvascular complications which is partly due to inflammation. Inflammation is strongly associated with both the secretory function of beta cells and insulin resistance. ${ }^{12}$ Circulating inflammatory molecules can decrease beta cell functions directly by secretory dysfunction or 
uncontrolled apoptosis. ${ }^{12}$ As a result, glucotoxicity and lipotoxicity occur and cause an enhanced inflammatory process. ${ }^{12}$ It is well known that inflammation is associated with an increased WBC count. Elevated levels of NLR have been found in diabetes and diabetic nephropathy, ${ }^{19,20}$ whereas elevated MLR levels have been found in diabetic retinopathy. ${ }^{17}$

The study conducted by Yue et al. to assess the use of the MLR to predict diabetic retinopathy showed that the MLR was increased in patients with diabetic retinopathy. ${ }^{17}$ However, the study did not compare MLR in controlled and uncontrolled T2DM. In our study, the difference in MLR while comparing controlled and uncontrolled T2DM was found to be statistically insignificant. There was a weak positive correlation found between glycated $\mathrm{Hb}$ levels and MLR values, but this result was also statistically insignificant.

A study conducted by Demirtas et al. to assess the association of hematological indices with diabetes, impaired glucose regulation, and microvascular complications of diabetes. ${ }^{12}$ The study found that there was a statistically significant difference between healthy, control groups, and diabetic groups with respect to NLR. This study shows that there is an increase in inflammatory markers in T2DM. Another study conducted by Hussain et al. on NLR: a good assessment tool of glycemic control in T2DM patients showed that increased NLR values were associated with elevated $\mathrm{HbA1c}$ levels and poor glycemic control in patients of T2DM. ${ }^{28}$ Although there is only a weak positive correlation present between glycated $\mathrm{Hb}$ levels and the ratios, the results of our study showed a significant difference in NLR while comparing controlled and uncontrolled diabetics. Studies comparing $\mathrm{HbA1c}$ with inflammatory markers in controlled and uncontrolled diabetics have been promising and this outcome should increase the scope of using these ratios in the evaluation of T2DM.

Our study has some limitations. First, a small sample size from a single institution may not represent the general population. Second, we cannot determine a cause and effect relationship due to the cross-sectional nature of our study.

\section{References}

1. Mathers $C D$, Loncar D. Projections of global mortality and burden of disease from 2002 to 2030. PLoS Med 2006;3(11):e442. DOI: 10.1371/ journal.pmed.0030442.

2. Shoelson $\mathrm{SE}$, Lee J, Goldfine AB. Inflammation, and insulin resistance. J Clin Invest 2006;116(7):1793-1801. DOI: 10.1172/JCI29069.

3. Lontchi-Yimagou E, Sobngwi E, Matsha TE, et al. Diabetes mellitus and inflammation. Curr Diab Rep 2013;13(3):435. DOI: 10.1007/s11892013-0375-y.

4. Ziaee A, Ghorbani A, Kalbasi S, et al. Association of hematological indices with prediabetes: a cross-sectional study. Electron Physician 2017;9(9):5206-5211. . Published 2017 10.19082/5206.

5. Luo Y, She DL, Xiong H, et al. Pretreatment neutrophil to lymphocyte ratio as a prognostic predictor of urologic tumors: a systematic review and meta-analysis. Medicine (Baltimore) 2015;94(40):e1670. DOI: 10.1097/MD.0000000000001670.

6. Gunduz S, Mutlu H, Tural D, et al. Platelet to lymphocyte ratio as a new prognostic for patients with metastatic renal cell cancer. Asia Pac J Clin Oncol 2015;11(4):288-292. DOI: 10.1111/ajco.12358.

7. Gu L, Ma X, Wang $L$, et al. Prognostic value of a systemic inflammatory response index in metastatic renal cell carcinoma and construction of a predictive model. Oncotarget 2016;8:52094-52103. DOI: 10.18632/ oncotarget.10626.

8. Semeniuk-Wojtaś A, Lubas A, Stec R, et al. Neutrophil-to-lymphocyte ratio, platelet-to-lymphocyte ratio, and C-reactive protein as new and simple prognostic factors in patients with metastatic renal cell cancer treated with tyrosine kinase inhibitors: a systemic review and meta-analysis. Clin Genitourin Cancer 2018;16(3):e685-e693. DOI: 10.1016/j.clgc.2018.01.010 ISSN 1558-7673.

9. Akyel A, Yayla Ç, Erat M, et al. Neutrophil-to-lymphocyte ratio predicts hemodynamic significance of coronary artery stenosis. Anatol J Cardiol 2015;15(12):1002-1007. DOI: 10.5152/akd.2015.5909.

10. Oylumlu M, Yıldız A, Oylumlu M, et al. Platelet-to-lymphocyte ratio is a predictor of in-hospital mortality patients with acute coronary syndrome. Anatol J Cardiol 2015;15(4):277-283. DOI: 10.5152/ akd.2014.5366.

11. Warimwe GM, Fletcher $\mathrm{HA}$, Olotu $\mathrm{A}$, et al. Peripheral blood monocyteto-lymphocyte ratio at study enrollment predicts efficacy of the RTS, $S$ malaria vaccine: analysis of pooled phase II clinical trial data. BMC Med 2013;11:184. DOI: 10.1186/1741-7015-11-184.

12. Demirtas L, Degirmenci H, Akbas EM, et al. Association of hematological indicies with diabetes, impaired glucose regulation and microvascular complications of diabetes. Int J Clin Exp Med 2015;8(7):11420-11427.

13. Akbas EM, Demirtas L, Ozcicek A, et al. Association of epicardial adipose tissue, neutrophil-to-lymphocyte ratio and platelet-tolymphocyte ratio with diabetic nephropathy. Int J Clin Exp Med 2014;7(7):1794-1801.

14. Ciray H, Aksoy AH, Ulu N, et al. Nephropathy, but not angiographically proven retinopathy, is associated with neutrophil to lymphocyte ratio in patients with type II diabetes. Exp Clin Endocrinol Diabetes 2015;123(5):267-271. DOI: 10.1055/s-0035-1547257.

15. Ulu SM, Dogan M, Ahsen A, et al. Neutrophil-to-lymphocyte ratio as a quick and reliable predictive marker to diagnose the severity of diabetic retinopathy. Diabetes Technol Ther 2013;15(11):942-947. DOI: 10.1089/dia.2013.0097.

16. Wang RT, Zhang JR, Li Y, et al. Neutrophil-lymphocyte ratio is associated with arterial stiffness in diabetic retinopathy in type II diabetes. J Diabetes Complicat 2015;29(2):245-249. DOI: 10.1016/j. jdiacomp.2014.11.006.

17. Yue S, Zhang J, Wu J, et al. Use of the monocyte-to-lymphocyte ratio to predict diabetic retinopathy. Int J Environ Res Public Health 2015;12(8):10009-10019. DOI: 10.3390/ijerph120810009.

18. Onalan E, Gozel N, Donder E. Can hematological parameters in type Il diabetes predict microvascular complication development? Pak J Med Sci 2019;35(6):1511-1515. DOI: 10.12669/pjms.35.6.1150.

19. Chung FM, Tsai JC, Chang DM, et al. Peripheral total and differential leukocyte count in diabetic nephropathy: the relationship of plasma leptin to leukocytosis. Diabetes Care 2005;28(7):1710-1717. DOI: 10.2337/diacare.28.7.1710.

20. Dada OA, Uche E, Akinbami A, et al. The relationship between red blood cell distribution width and blood pressure in patients with type II diabetes mellitus in Lagos, Nigeria. J Blood Med 2014;5:185-189. DOI: 10.2147/JBM.S67989.

21. Tesfamariam B, Cohen RA. Free radicals mediate endothelial cell dysfunction caused by elevated glucose. Am J Physiol 1992;263(2 Pt 2):H321-H326. DOI: 10.1152/ajpheart.1992.263.2.H321.

22. Yang AP, Liu JP, Tao WQ, et al. The diagnostic and predictive role of NLR, d-NLR and PLR in COVID-19 patients. Int Immunopharmacol 2020;84:106504. DOI: 10.1016/j.intimp.2020.106504.

23. Akinci Ozyurek B, Sahin Ozdemirel T, Buyukyaylaci Ozden S, et al. Prognostic value of the neutrophil to lymphocyte ratio (NLR) in lung cancer cases. Asian Pac J Cancer Prev 2017;18(5):1417-1421. Published 2017 May 1 10.22034/APJCP.2017.18.5.1417.

24. Onsrud $M$, Thorsby E. Influence of in vivo hydrocortisone on some human blood lymphocyte subpopulations. I. Effect on natural killer cell activity. Scand J Immunol 1981;13(6):573-579. DOI: 10.1111/j.1365-3083.1981.tb00171.x. https://www.ncbi.nlm.nih.gov/ pubmed/7313552. 
25. Ye G, Chen $\mathrm{Q}$, Chen $\mathrm{X}$, et al. The prognostic role of platelet-tolymphocyte ratio in patients with acute heart failure: a cohort study. Sci Rep 2019;9(1):10639. DOI: 10.1038/s41598-019-47143-2.

26. Stojkovic Lalosevic M, Pavlovic Markovic A, Stankovic S, et al. Combined diagnostic efficacy of neutrophil-to-lymphocyte ratio (NLR), platelet-to-lymphocyte ratio (PLR), and mean platelet volume (MPV) as biomarkers of systemic inflammation in the diagnosis of colorectal cancer. Dis Markers 2019;2019:6036979. DOI: 10.1155/2019/6036979.

27. Chen $\mathrm{H}$, Li M, Liu L, et al. Monocyte/lymphocyte ratio is related to the severity of coronary artery disease and clinical outcome in patients with non-ST-elevation myocardial infarction. Medicine (Baltimore) 2019;98(26):e16267. DOI: 10.1097/MD.000000000 0016267.

28. Hussain M, Babar MZM, Akhtar L, et al. Neutrophil lymphocyte ratio (NLR): a well assessment tool of glycemic control in type II diabetic patients. Pak J Med Sci 2017;33(6):1366-1370. DOI: 10.12669/ pjms.336.12900.

29. Nguyen DV, Shaw LC, Grant MB. Inflammation in the pathogenesis of microvascular complications in diabetes. Front Endocrinol (Lausanne) 2012;3:170.DOI: 10.3389/fendo.2012.00170Published 2012 Dec 21. 